\title{
LOW-LEVEL STELLAR VARIABILITY
}

\author{
M. GRENON \\ Geneva Observatory, CH-1290 Sauverny, Switzerland
}

\begin{abstract}
The capability of ground-based photometry to detect and monitor low-level stellar variations is discussed in the particular case of amplitudes in the range of few magnitudes to few percent. The teclunical and atmospherical constraints are briefly recalled. The variability level of the so-called constant stars is discussed as function of the spectral type, the luminosity class and the effective temperature. The photometric aspects of the Hipparcos mission are presented here since this satellite allows a systematic detection of microvariables and the obtention of light curves across the whole HR-diagram with an unprecedented efficiency.
\end{abstract}

\section{INTRODUCTION}

A global description of the variability in the HR-diagram at the milimagnitude level is still missing. In the following, we intend to describe what is now known from ground based photometry and what the expectations from space photometry with the Hipparcos satellite are. The emphasis will be put on variability detection and light curve obtention as a by-products of ground and space programs.

For specific objects, differential photometry allows precise determinations of the amplitudes at the sub-milimagnitude level provided that the periods are short with respect of the night duration. Multi-site observations partly overcome this difficulty. For longer period stars, the variability characterisation results from absolute photometry, the detection threshold is then no better than a few milimagnitudes but there is no more limitation regarding the period which may range from few minutes to several years.

A nomenclature problem arises for the classification of variables according to their amplitude range. Classical variables show amplitudes (A) which are of the order of a magnitude or a substantial fraction of it, the term of microvariable is currently and somewhat improperly applied to variables with amplitudes in the range of few percents. What should be the designation for variables with $A$ in the range of few $10^{-3}$ or $10^{-4} \mathrm{mag}$ ? Whatever will be the accepted designation, we will investigate here the variability of so-called microvariable and constant stars. 


\section{THE DETECTION THRESHOLD}

For many years, ground-based observers considered it to be fashionable to produce colours and magnitudes with an accuracy not better than a few percent. The adopted integration times, the number of comparison stars, the thermal control of the instruments and adopted reduction procedure, lead ultimately to errors, in the case of the classical UBV photometry, of $0.02 \mathrm{mag}$ on (B-V) and $0.03 \mathrm{mag}$ on $\mathrm{V}$.

As a matter of fact, these errors may be reduced by one order of magnitude, provided a number of precautions are taken for the construction of the photometers and for the data collection and reduction. When observing from the ground, the most limiting factor, magnitude independent, is the scintillation. To reach to $0.005 \mathrm{mag}$ level requires integration times not shorter than $10 \mathrm{sec}$ onds for a $70 \mathrm{~cm}$ telescope. Too often integrations are stopped when the photon noise is below a specified level. The atmospherical extinction changes seriously limit the photometric accuracy. During typical photometric nights, the visual extinction may vary by $0.02-0.03$ at the Zenith. After events like the El Chichon or more recently the Pinatubo eruption, the atmospherical extinction becomes anisotropic and variable. As an example, four months after the Pinatubo eruption, up to 8 comparison stars per hour were necessary to monitor the extinction at the $0.002 \mathrm{mag}$ level. The thermal control of the detector is critical too. Thermal drifts induces sensitivity changes of the detectors and pass-bands shifts of the combination filter plus detector. A temperature stabilisation of $\pm 0.1^{\circ} \mathrm{C}$ ensures a gain stability of \pm 0.4 milimagnitude. Photon-noise stars to be significant only for rather faint stars or extreme colour stars. The ultimate accuracy is also dependent on the stability of comparison stars themselves. Too often lists of standards are contaminated by microvariables (up to $40 \%$ in a recent VRI standard) and observers are tempted to use stars with a large number of observations as comparison, whereas it is precisely because they were suspected variables that they receive more attention.

Provided that the above conditions are fulfilled, it is possible to achieve absolute photometry at the $0.002-0.003 \mathrm{mag}$ level with a one meter telescope. For more details on accuracy photometry see e.g. Harvey (1988) and Young (1984).

\section{THE GENEVA PHOTOMETRY ACCURACY}

Since the installation of the Swiss telescope at La Silla in 1976 and the use of a new generation photometer, the accuracy of photometric data in the Geneva system was seriously improved, because the technical improvements and the quality of the Chilean skies. The observational strategy is to observe a star twice at a one month's interval approximately, and then for a third time one year later. Colours and magnitudes are means over 3 to 12 minutes observations. This procedure allows a detection, if not a description, of the short and long-term variables.

The distributions of residuals on $\mathrm{V}$ magnitude is given Fig. I. The mode of the distribution is in the range 2 to 4 milimagnitudes. Stars with larger $\sigma \mathrm{V}$ are either faint stars or microvariables. Bright stars are suspected as microvariables 


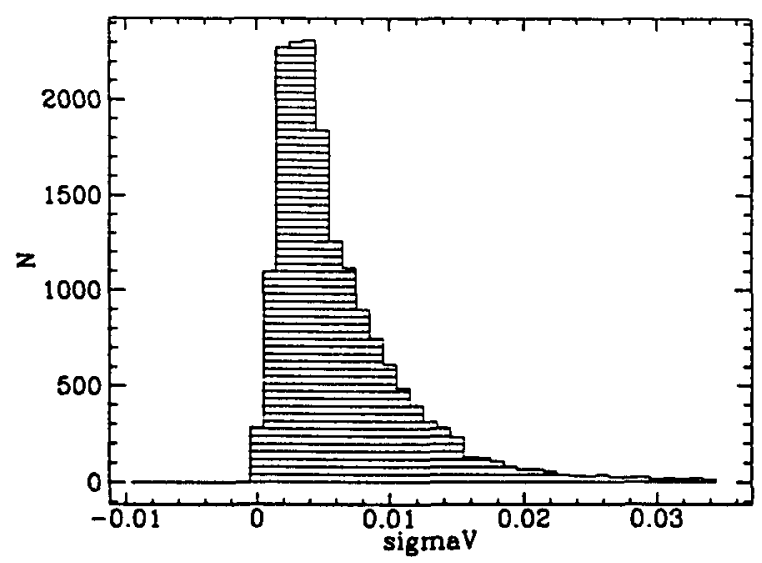

FIGURE I The distribution of residuals on V mag for 29000 stars with three or more measurements in Geneva photometry.

if $\sigma \mathrm{V}>0.006$ mag. Possible variables are almost systematically re-observed to confirm their status and a number of 6-10 observations are generally performed.

The photometric catalogue of Geneva contains now 40000 stars and among them 29000 have been observed at least three times. Since all spectral types and luminosities are represented, a global investigation of the stellar stability may be attempted across the whole HR-diagram.

\section{THE RELATION SPECTRAL-TYPE-AMPLITUDE}

Since early-type and luminous stars are often heavily reddened, a straightforward way to reveal the instability domains as a function of the temperature, is to plot the residuals in $\mathrm{V}$ versus the spectral type.

Figure II shows $\sigma \mathrm{V}$ as a function of the digitised type irrespective to the luminosity class. Non variable stars show $\sigma \mathrm{V}=0.0048 \mathrm{mag}$ and a mean standard deviation of the colours of $0.00371 \mathrm{mag}$. The classical instability strip is centred on F3 for main sequence stars and extends towards lower temperature with increasing luminosity and amplitude. Stars earlier than B1 appear quite stable, with the exception of WR stars which are all microvariables. The most striking feature is the B1-B2 range with many stars showing standard deviation between .04 and $.17 \mathrm{mag}$. This $\kappa$-driven instability concerns a very narrow range of $T_{\text {eff }}$ if one takes into account the uncertainty on the spectral type itself. The domain B8-A0 contains the magnetic and peculiar Ap, Bp stars. Their amplitudes in V are half those of $\mathrm{B} 1-\mathrm{B} 2$ stars. The noise increases at $\mathrm{K} 0$ is conspicuous as well as that of $\mathrm{M}$ stars with $\sigma \mathrm{V}$ increasing with advancing $\mathrm{M}$ subtype.

When the standard deviation on colours $(\sigma \mathrm{col})$ is considered, see Fig. II, the behaviour as a function of the spectral type is fairly different. At given $\sigma \mathrm{V}$, the corresponding $\sigma$ col decreases with increasing $\mathrm{T}_{\text {eff }}$; this effect is a consequence of the loss of sensitivity to $\mathrm{T}_{\mathrm{eff}}$ of the colour indices towards high $\mathrm{T}_{\mathrm{eff}}$. 


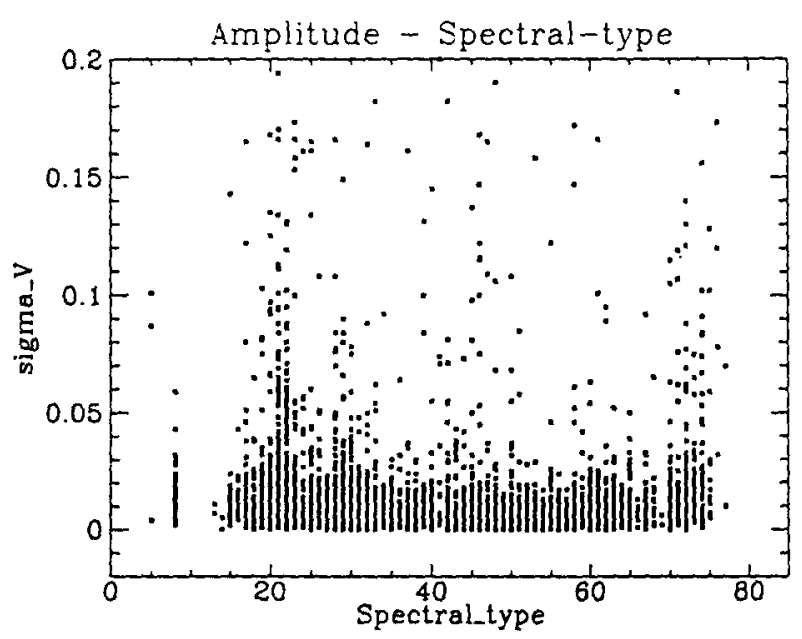

FIGURE II The distribution of the standard deviation in $\mathrm{V}$ as function of the spectral type. Coding of types: $08=\mathrm{WR} ; 15=05 ; 20=\mathrm{B} 0 ; 30=\mathrm{A} 0$; $40=\mathrm{F} 0 ; 50=\mathrm{G} 0 ; 60=\mathrm{G} 0 ; 70=\mathrm{M} 0$.

\section{THE RELATION LUMINOSITY-TEMPERATURE-AMPLITUDE}

If the stellar plysical properties are homogeneous in a given range of spectral type and luminosity class, it is equivalent to derive the mean variability level from large sets of observations on few representative stars or from a large collection of stars with few measurement per star. The number of stars in the Geneva catalogue with $n>2$ observations per star permits the second alternative.

For the discretisation in terms of luminosity, the following classes are retained: Ia0-Ia, Ib,II, II-III, III, III-IV, IV, IV-V, V, V-VI and VI. In terms of spectral type, bins of one to three sub-types are considered. The number of stars per box is very variable but high for the main sequence with a maximum of 880 for B8-B9V stars. The minimum standard deviation is observed for late $F$ and early $G$ main sequence stars and is equal to $0.0048 \mathrm{mag}$. This value is adopted as the measurement error. For a given box, the intrinsic stellar standard deviation on $\mathrm{V}$ is computed as follows:

$$
\sigma V_{i}=\left(\sigma V_{o b s}^{2}-0.0048^{2}\right)^{1 / 2}
$$

where $\sigma \mathrm{V}_{\mathrm{obs}}$ is the mean observed standard deviation in that box. In order to minimise the contamination by eclipsing binaries, all stars with $\sigma \mathrm{V}>0.2 \mathrm{mag}$ or $\sigma \mathrm{col}>0.1$ are rejected from the samples. The distribution of variability domains in Fig. IV, is then that of low amplitude and "constant" stars; the mean amplitude is largely underestimated for stars inside the instability strip and for late $M$ giants.

The high stability areas in the HR-diagram are: the F2-G5 main sequence, the F1-G9 class IV-V, the G0-G9 subgiants and the III-IV first giant branch. The bright G2-K2 giants of class II are remarkably stable too with a mean rms $<5$ mmag. On the blue side of the instability strip, the evolved B5-A6 stars are very stable provided they are less luminous than the $\mathrm{Ia}, \mathrm{Ib}$ supergiants. 


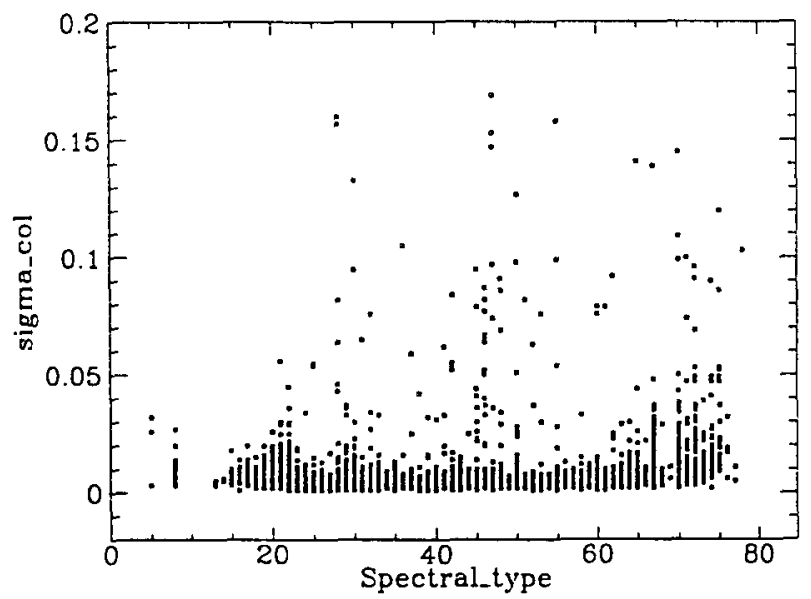

FIGURE III The distribution of the standard deviation on Geneva colours as function of the spectral type. Codes for the type as in Fig. III.

The $\kappa$-mechanism is quite efficient for B1-B2 dwarfs and giants with typical rms of 15 to 40 mmag. Its efficiency tends to disappear at higher luminosity or to be displaced to lower temperatures. All luminous supergiants are microvariables or variables, especially in the vicinity of the instability strip.

The microvariability level of A5-G5 class III giants is of the order of 7 mmag outside the instability strip. The variability is partly due to the presence of a higher than normal proportion of double stars with composite spectra.

On the red side of the instability strip, there is a gentle increase of the variability with the temperature decrease down to the early M stars. At M1-M2, there is a sudden increase corresponding to the development of $\mathrm{TiO}$ absorption bands. The old mild metal poor stars and the population II subdwarfs seem less stable than main sequence population I of the same mass.

\section{THE LATE TYPE STARS VARIABILITY}

For most red dwarfs and part of supergiants, an MK classification is not available. For stars with a negligible interstellar reddening, the calibrations of the Geneva photometry provide estimates of $\mathrm{Mv}, \mathrm{T}_{\mathrm{eff}},[\mathrm{M} / \mathrm{H}]$ or $\mathrm{I}_{\mathrm{TiO}}$ with a precision sufficient for an unambiguous attribution to the various branches in the HR-diagram.

The HR-diagram of Fig. V results from an automatic photometric classification of field stars and contains 7915 stars found to lie within $150 \mathrm{pc}$ where the reddening may be neglected. Stars below the main sequence and the red giant branch have composite spectra or colours and spurious Mv. M giants are not plotted here since their absolute magnitudes are still undefined. 


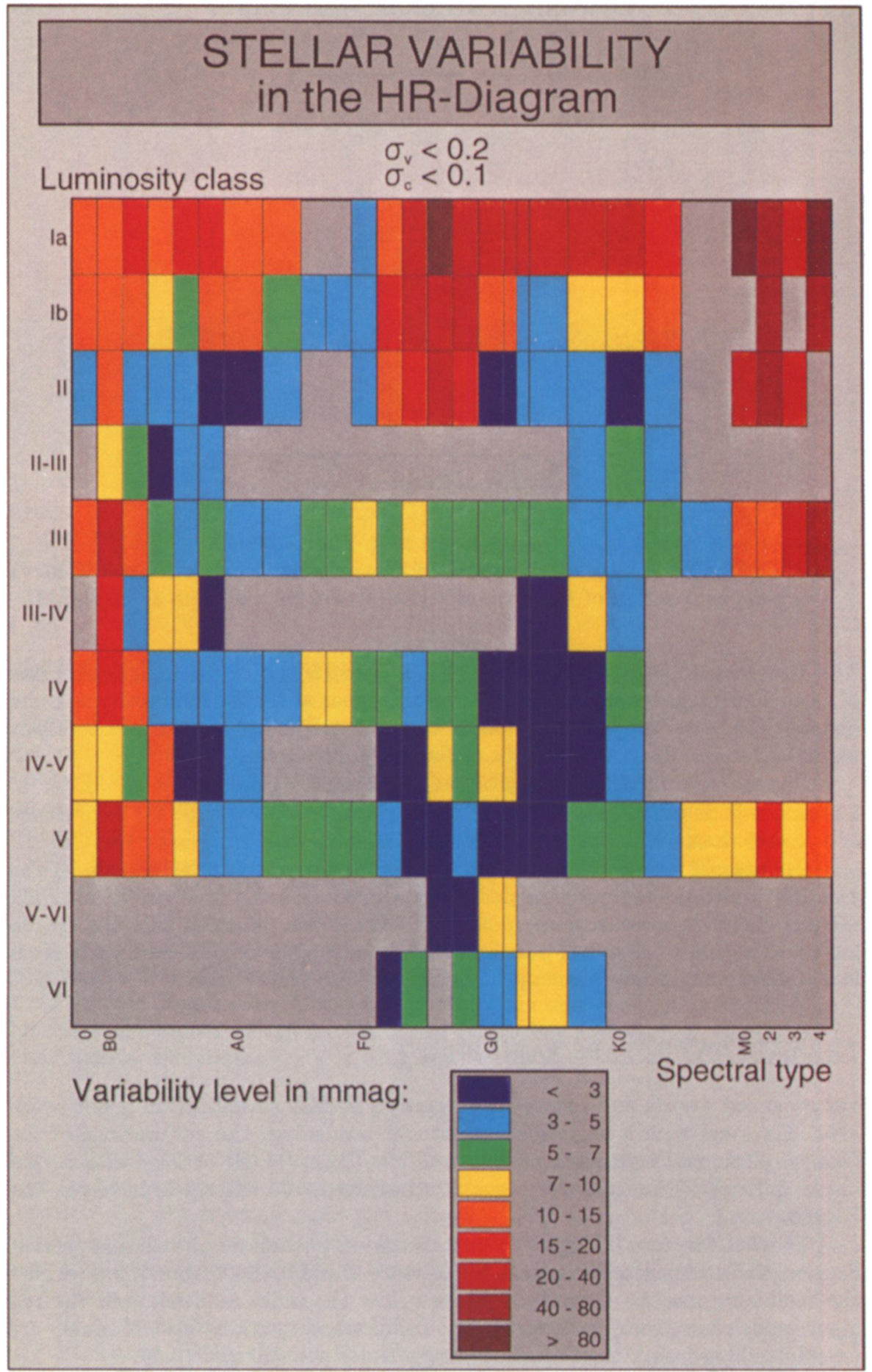

FIGURE IV The intrinsic low level variability in the HR-diagram. Stars with $\sigma \mathrm{V}>0.2$ or $\sigma \mathrm{col}>0.1$ are excluded. The diagram is based on the properties of 29000 stars with 3 or more measurements in Geneva photometry. 


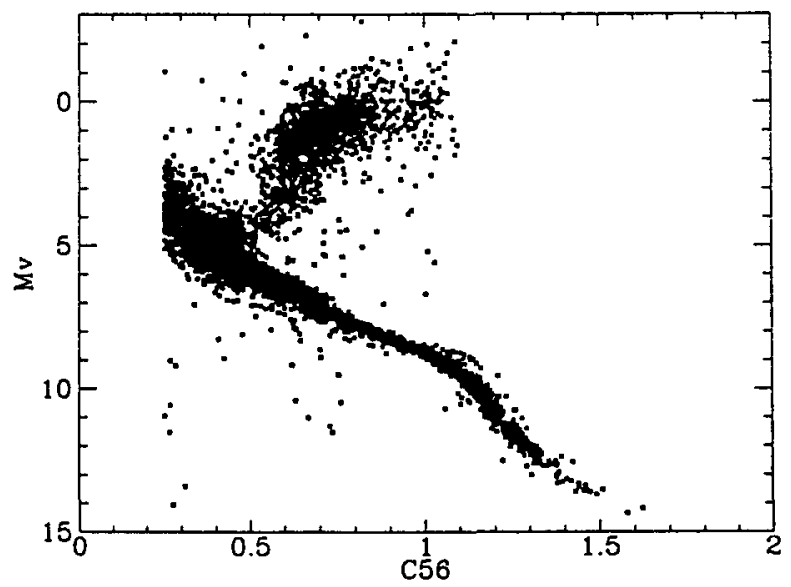

FIGURE V The HR-diagram of 7915 field stars within 150 pc from Geneva calibrations. The blue limit of C56 index (三 (B2-V1)) corresponds to the F5 type.

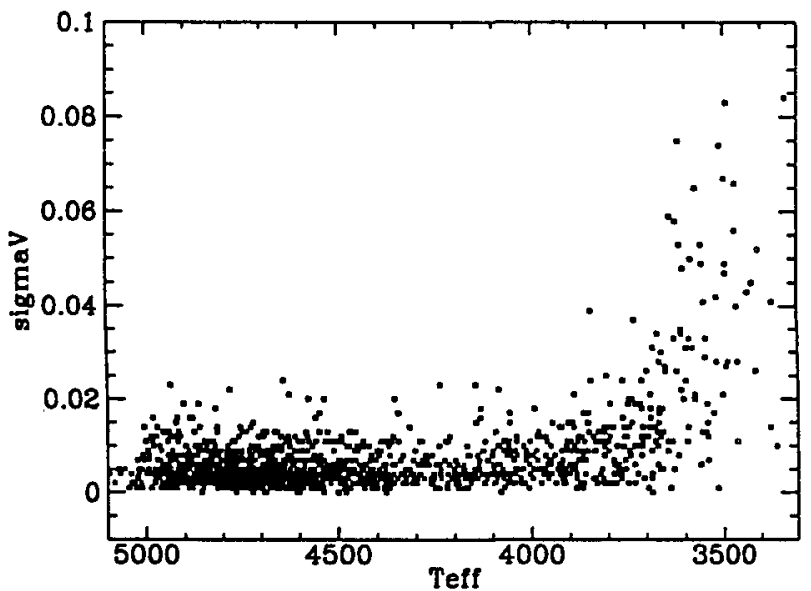

FIGURE VI The distribution of $\sigma \mathrm{V}$ for 1536 red giants with $\mathrm{Mv}$ in the range -2 to +2 and $d<150 \mathrm{pc}$. 
The distribution of $\sigma \mathrm{V}$ as a function of $\mathrm{T}_{\mathrm{eff}}$ is given in Fig. VI for the red giants. The minimum amplitude occurs in the temperature interval 4500-4700 ${ }^{\circ} \mathrm{K}$. The slight variability increase in the range $4800-5100^{\circ} \mathrm{K}$ is probably due to the higher fraction of binaries at the left of the first giant branch and of the clump, not distinguished here. The increase of $\sigma \mathrm{V}$ below $4000^{\circ} \mathrm{K}$ is fairly proportional to the amount of $\mathrm{TiO}$ absorption. The intrinsic dispersion $\sigma \mathrm{V}_{\mathrm{i}}$ is given in Table I for red giants with $\sigma_{\mathrm{V}}<0.2$ and $\sigma_{\text {col }}<0.1$ mag.

TABLE I The intrinsic standard deviation in $V$ for red giants, in units of $10^{-4}$ magnitude with the number $\mathrm{N}$ of stars in each interval of $T_{\text {eff }}$.

\begin{tabular}{crr}
$\mathrm{T}_{\text {eff }}$ & \multicolumn{1}{c}{$\sigma \mathrm{V}_{\mathrm{i}} \pm \mathrm{pe}$} & $\mathrm{N}$ \\
\hline $4900-5000$ & $35 \pm 4$ & 130 \\
$4800-4900$ & $23 \pm 3$ & 170 \\
$4700-4800$ & $0 \pm 2$ & 227 \\
$4600-4700$ & $6 \pm 2$ & 210 \\
$4500-4600$ & $10 \pm 3$ & 147 \\
$4400-4500$ & $5 \pm 3$ & 100 \\
$4300-4400$ & $24 \pm 5$ & 61 \\
$4200-4300$ & $27 \pm 5$ & 49 \\
$4100-4200$ & $33 \pm 5$ & 63 \\
$4000-4100$ & $41 \pm 5$ & 63 \\
$3900-4000$ & $53 \pm 5$ & 63 \\
$3800-3900$ & $82 \pm 9$ & 61 \\
$3700-3800$ & $99 \pm 10$ & 53 \\
$3600-3700$ & $193 \pm 23$ & 50 \\
$3500-3600$ & $272 \pm 34$ & 28 \\
\hline \hline
\end{tabular}

A large body of main-sequence stars was for several purposes observed in Geneva photometry. They were selected according to a minimum parallax, a limiting magnitude and, most frequently, to a minimum proper-motion. The sample of 2870 stars with $\mathrm{Mv}>5.0$ is representative of the old disc and halo. Nearby stars may be partially younger. The intrinsic variability is fairly constant in the temperature range $4200^{\circ} \mathrm{K}-5200^{\circ} \mathrm{K}$, with rms of the order of 4 to 6 mmag. A discontinuity seems to appear around $4200^{\circ} \mathrm{K}$ at the location of the Mazzitelligap (1972) sometimes referred as the Wielen gap (1974). Between 3500 and $4200^{\circ} \mathrm{K}$ the typical rms is $8 \mathrm{mmag}$. The increase at lower temperatures is partly due to a photon-noise effect on faint late $M$ stars, not corrected here.

The relation between the intrinsic $\sigma \mathrm{V}_{\mathrm{i}}$ and the effective temperature is given in Fig. VII for the low main sequence, parallel with the number of stars per interval of temperature.

The change in the temperature-luminosity relation in the main sequence with its associated change of variability level occurs at a temperature corresponding to the spectral type K7V. 


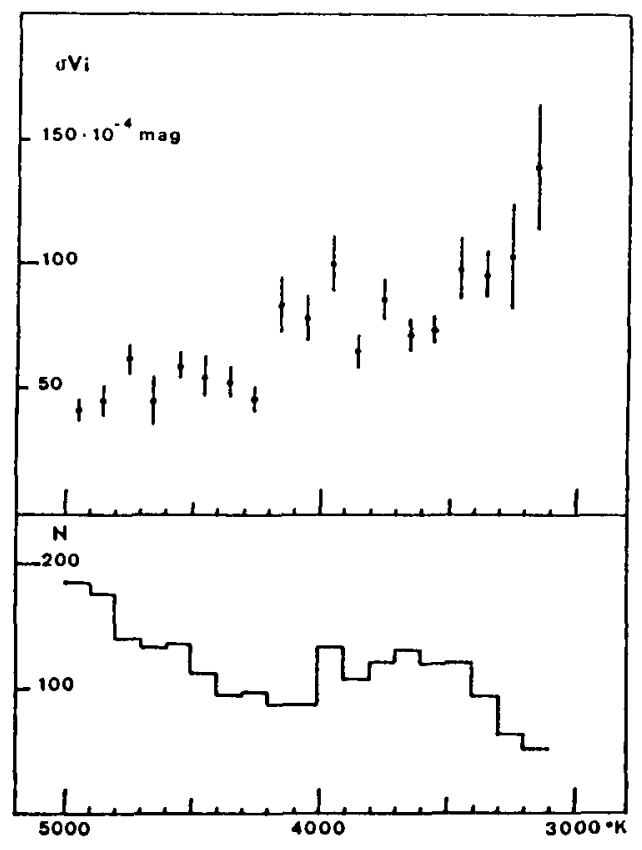

FIGURE VII The relation $\sigma \mathrm{V}_{i}$ versus $\mathrm{T}_{\text {eff }}$ for $2200 \mathrm{~K}$ and $\mathrm{M}$ dwarfs, within $150 \mathrm{pc}$ and with $\mathrm{N}>2$ measurements (top). Below, the number of stars per $T_{\text {eff }}$ interval shows the presence of the Mazzitelli-gap at 4000 $4200^{\circ} \mathrm{K}$.

A physical study of the variability along the main sequence would imply to consider other indicators of the stellar activity as the variability in the UV, the effects of star spots through the luminosity changes and the TiO intensity variations. Also, it is necessary to distinguish single stars from binaries and to evaluate the age and overall metallicity effects. These aspects are beyond the scope of this presentation.

For a discussion of the physical processes responsible for the microvariability in late -type stars, see Gray, 1991.

\section{LIGHT CURVES OF LOW AMPLITUDE VARIABLES}

The luminosity changes of low level variables are often far from being simple. They may be erratic , wind driven, in the case of early type luminous stars, semiperiodic with phase shifts when produced by transit of groups of star spots, or multiperiodic in the case of low amplitude pulsating B stars.

The characterisation of the variability requires frequently several hundred measurements collected over long intervals of time, when the searched effects are close to the detection limit. As an example, the eclipsing binary RZ Eri was monitored for 11 years before Burki et al. (1992) were in the position to disentangle the reflection from the starspot effect in the secondary component of this system. 


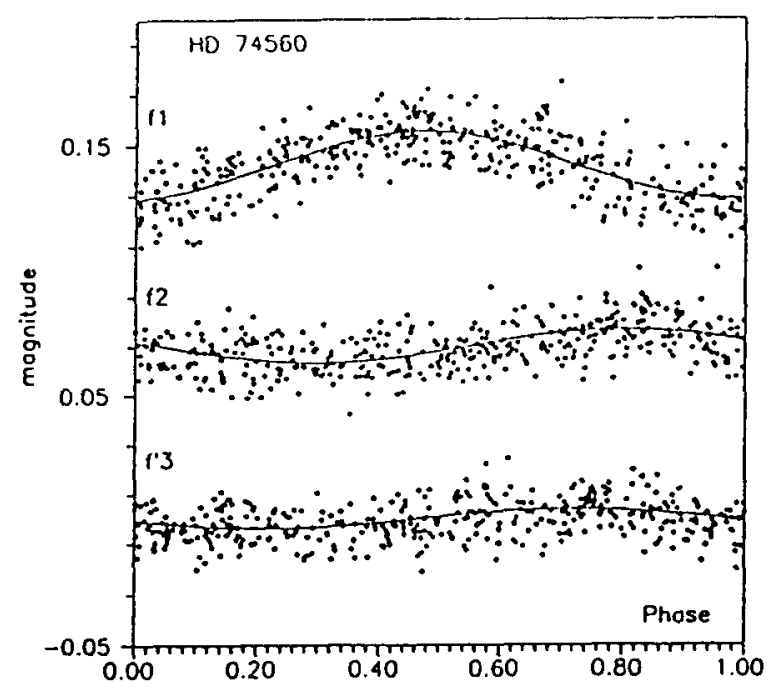

FIGURE VIII Phase diagrams for the visual magnitude data of HD 74560 by Waelkens (1991).

The analysis of slowly pulsating $B$ type stars was undertaken by the Leuven group for a small number of candidates. The phase diagram for the visual magnitude data of HD 74 560, by Walkens (1991) is a good illustration, cf. Fig. VIII, of the ground-based photometry capability and limitations. No less than 408 observations, collected between 1976 and 1989, were necessary to discover the three non-radial modes with $\mathrm{P}=1.551,1.645$ and 1.737 days and $\mathrm{A}=0.027,0.013$ and $0.008 \mathrm{mag}$ respectively.

A second example is the light-curve of a multiperiodic B8V star shown in Fig. IX. This star studied by North (1992) has four determined periods up to now, i.e. $\mathrm{P} 1=0.8044, \mathrm{P} 2=0.847, \mathrm{P} 3=0.9032$ and $\mathrm{P} 4=0.9591 \mathrm{~d}$. Even with a set of $\mathbf{4 3 5}$ measurements, there remain mismatches between the observed lightcurve and that computed from the provisional periods, phases and amplitudes.

Similar problems arise for the description of the stellar cycles among late $K$ and $M$ dwarfs. The size of the amplitudes, the number of necessary measurements, clearly indicate that the limits of absolute ground based photometry are met. 


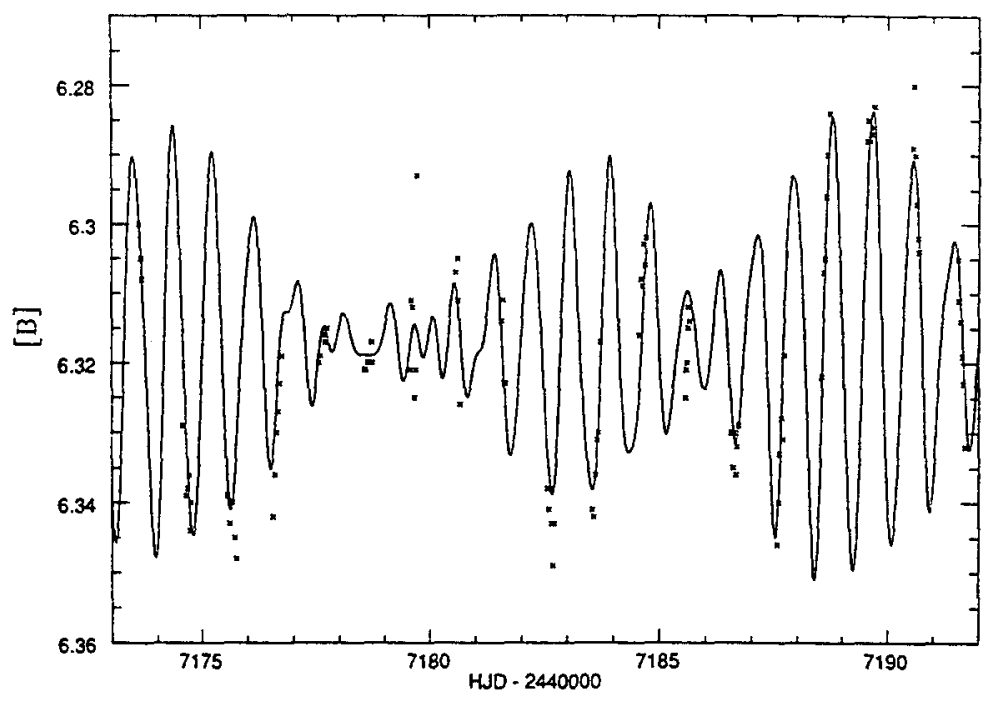

FIGURE IX The light curve in B magnitude of the multiperiodic B8V star, HD 37151 by North (1992).

The number of cases which may be studied in such detail is necessarily very limited. This is why mass production of accurate magnitudes from the space is so attractive.

\section{THE HIPPARCOS PHOTOMETRIC SURVEYS}

During the development of the satellite, the detection chains were modified in order to optimise the photometric outputs. An accurate calibration procedure was set up to allow high accuracy photometry with the main mission detector. The broad band, $\mathrm{H}_{\mathrm{p}}$, extends from 380 to $900 \mathrm{~nm}$ and is defined in order to optimise the signal-to-noise ratio and hence the detection of luminosity changes (see Grenon, 1992).

The star mapper chains were equipped with blue and visual filters denoted $\mathrm{B}_{\mathrm{T}}$ and $\mathrm{V}_{\mathrm{T}}$. Their effective wavelengths and widths optimise both the satellite attitude determination and the accuracy on star colours.

With the main-mission detector, the 118000 program stars are with regularity observed throughout the mission. The list of monitored stars is published in the Hipparcos Input Catalogue by Turon et al. (1992).

All stars brighter than $B_{T}$ or $V_{T}=10.5$ are systematically observed with the Tycho chains. They belong to an Input Catalogue of $2 \cdot 10^{6}$ stars computed from the Hubble Space Telescope Guide Star Catalogue (GSC). 
About 500000 objects will have $B_{T}$ and $V_{T}$ determined by the Tycho experiment. For about 500000 additional stars only one magnitude, and then no colour, will be determined.

\section{THE HIPPARCOS TIME-SAMPLING}

The sampling by the satellite is particular. Stars are observed 9 times during a Field of View (FOV) crossing of $20 \mathrm{sec}$ duration. Mean magnitudes, precision and epochs are stored after a detailed calibration process.

Stars are observed in two FOVs, separated by an angle of 58 degrees. Depending on its location on the sky, a given star is observed in groups of 4 to 6 transits. The time intervals between transits in the preceding and following FOVs is $21 \mathrm{~min}$, and $107 \mathrm{~min}$ between transits in the following and preceding FOVs.

According to the galactic latitude $\beta$, groups of transits are obtained at a rate of 9 to 25 groups per year. The maximum rate is for $\beta=45^{\circ}$. As the satellite precesses, about half of the program stars may occupy the node of the scanned great circle. Up to 50-60 transits may then be observed consecutively. A mean number of 200 observations per star is expected from the Hipparcos mission.

\section{THE ACCURACY ON $\mathrm{H}_{\mathrm{P}}, \mathrm{B}_{\mathrm{T}}, \mathrm{V}_{\mathrm{T}}$ MAGNITUDES}

Due to the absence of scintillation and in spite of the small telescope size, the photometric accuracy of the $H_{p}$ magnitude is very high. The accuracy is photonnoise limited and magnitude dependent. Tycho $\mathrm{B}_{\mathrm{T}}, \mathrm{V}_{\mathrm{T}}$ data are four times less accurate because of a shorter integration time and a higher background signal.

TABLE II The expected accuracy on $\mathrm{H}_{\mathrm{p}}$ as a function of the instrumental $H_{p}$-magnitude, and on $B_{T}$ and $\left(B_{T}-V_{T}\right)$ as function of the Tycho $B_{T}$ magnitude.

\begin{tabular}{|c|c|c|c|c|c|c|c|c|}
\hline \multicolumn{2}{|c|}{$\mathrm{H}_{\mathrm{P}}, \mathrm{B}_{\mathrm{T}}$} & : & 6 & 7 & 8 & 9 & 10 & 11 \\
\hline$\sigma_{\mathrm{H}_{\mathrm{p}}}$ & $\begin{array}{l}1 \text { transit } \\
5 \text { hours }\end{array}$ & : & $\begin{array}{l}.006 \\
.002\end{array}$ & $\begin{array}{l}.009 \\
.004\end{array}$ & $\begin{array}{l}.015 \\
.006\end{array}$ & $\begin{array}{l}.024 \\
.010\end{array}$ & $\begin{array}{l}.043 \\
.017\end{array}$ & $\begin{array}{l}.08 \\
.03\end{array}$ \\
\hline$\sigma_{\mathrm{B}_{\mathrm{T}}}$ & $\begin{array}{l}1 \text { transit } \\
5 \text { hours }\end{array}$ & : & $\begin{array}{l}.03 \\
.009\end{array}$ & $\begin{array}{l}.04 \\
.012\end{array}$ & $\begin{array}{l}.06 \\
.017\end{array}$ & $\begin{array}{l}.12 \\
.035\end{array}$ & $\begin{array}{l}.24 \\
.07\end{array}$ & $\begin{array}{l}.48 \\
.14\end{array}$ \\
\hline$\sigma_{\mathbf{B}_{\mathbf{T}}}$ & 5 hours & : & .013 & .017 & .024 & .049 & .10 & .20 \\
\hline
\end{tabular}

The typical accuracy for a given transit and for a group of transits over 5 hours is given in Table II. 


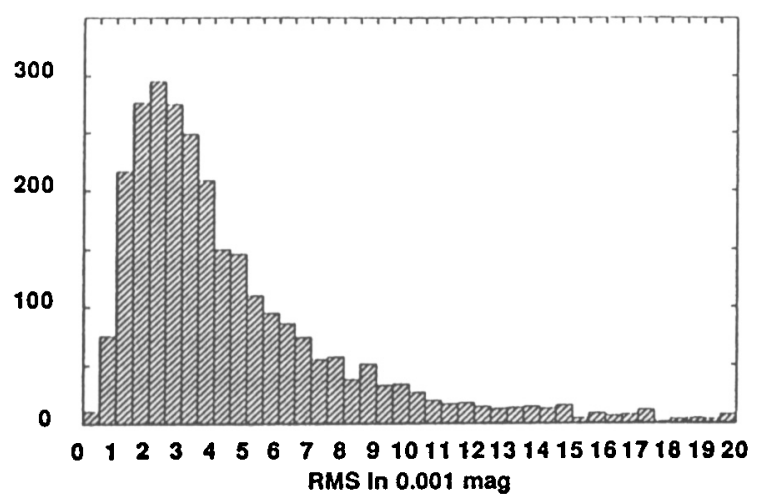

FIGURE X The residuals on $\mathrm{H}_{\mathrm{p}}$ magnitude, with one point per group of transits, from F. Mignard et al. (1992).

The irradiation by solar and cosmic particles reduces the transmission of the main mission optics at a rate of $+\mathbf{0 . 0 1 3} \mathbf{m a g}$ between two consecutive groups of transits. This transmission change is chromatic and, after a $4.5 \mathrm{y}$ mission, magnitudes of $(B-V)=0.0$ stars will be increased by +0.85 mag and those of the reddest stars by less than +0.20 mag. A continuous calibration, in-orbit, is performed by the data reduction consortia, using large sets of standard stars. The accuracy of the procedure is such that the zero point of the magnitude scale is daily defined with an error rate inferior to $0.001 \mathrm{mag}$. The distribution of residuals on $H_{p}$ magnitude (one point per group), see Fig. $X$, has a modal rms of 2 mmag. It demonstrates the stability of the main mission photometric system, in spite of the large transmission variations. The tail is due to the presence of faint stars and of microvariable stars. The precision of Hipparcos $\mathrm{H}_{\mathrm{p}}$ magnitudes, averaged over 5 hours, is higher than that of Geneva photometry for stars brighter than 6.5, similar to that of Strømgreen photometry by Olsen for $7.0 \mathrm{mag}$ stars and comparable to that of UBV photometry for $9.5 \mathrm{mag}$ stars.

No significant aging affect the Tycho $B_{T}$ and $V_{T}$ channels. The performances and properties of the Tycho system remain unaltered all along the mission.

\section{THE DETECTION OF VARIABLE STARS WITH HIPPARCOS}

Up to now, the minimum detection amplitude Amin was $0.2-0.3 \mathrm{mag}$ for surveys on photographic plates. From Tycho, the lowering of Amin to few percent will enormously increase the number of newly discovered or confirmed variables. The major gain from the Hipparcos mission will, because of its accuracy, come from the main-mission photometry. 
TABLE III The minimum detectable amplitude as function of the instrumental magnitude $\mathrm{H}_{\mathrm{p}}$ at a confidence level of $99 \%$.

\begin{tabular}{lccccc}
$\mathrm{H}_{\mathrm{p}}$ & $:$ & 6 & 8 & 10 & 12 \\
\hline $\mathrm{Amin}$ & $:$ & .007 & .017 & .048 & .20
\end{tabular}
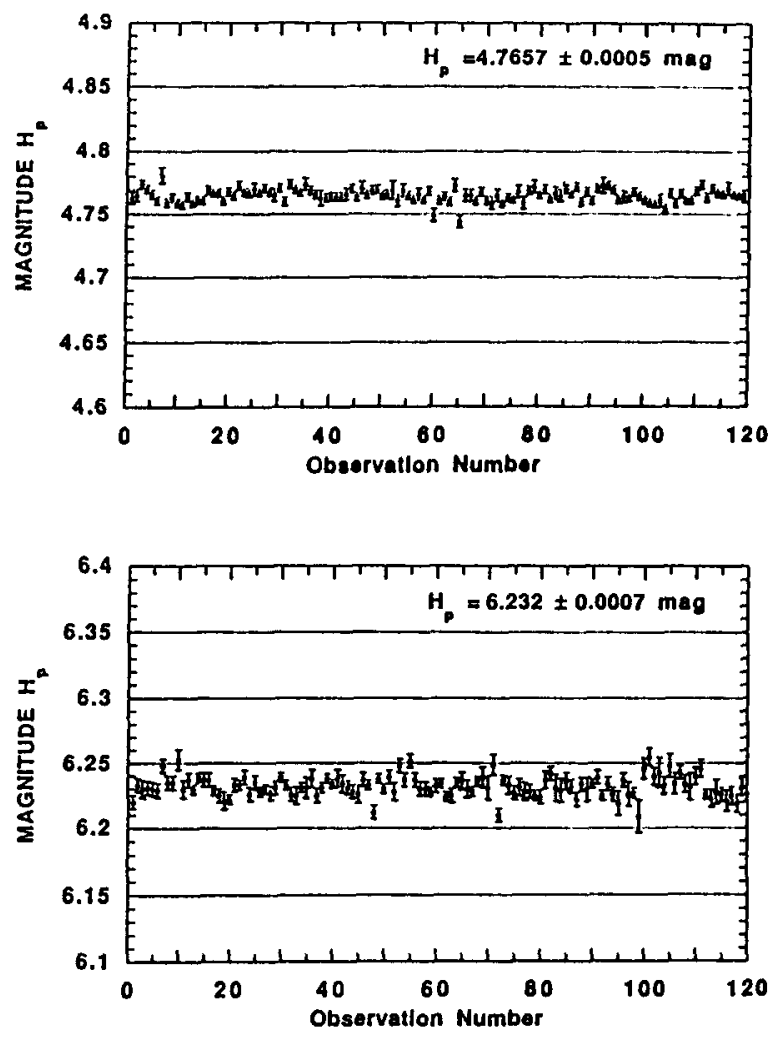

FIGURE XI Evolution of the instrumental magnitude in the case of a 4th and 6th magnitude star. From F. Mignard, FAST Consortium. 
In Tab. III, Amin is given as a function of $\mathrm{H}_{\mathrm{p}}$ in the case of a sinusoidal variation.

For bright stars, Amin is similar to that detectable with accurate groundbased photometry. The decisive advantage of Hipparcos photometry is the number of measurements per star, about 200, and the duration of the monitoring, i.e. 3.5-4.5 years. The time distribution of the observations is adequate for an unbiased detection of periods in the range of few minutes to several years. The Hipparcos sampling law is possibly not optimum for multiperiodic stars. It appears better suited for the detection of rotationally induced variability alike the $\mathrm{Bp}$ phenomenon or effects of stellar activity. Transits of spot groups of size similar to those of the Sun, are barely detectable for bright nearby stars.

In Fig. XI, examples of the evolution of the observed magnitude with the time are shown. Systematic departures from the mean value are possibly real. The stability of the photometric system is such that secular luminosity changes of the order of 0.001 to 0.002 magnitude per year are detectable. A few rapidly evolving stars may be discovered. More frequently, slow luminosity changes will result from opacity changes in circumstellar envelopes.

The detectable amplitude with Tycho was computed by Mauder \& Høg, 1987. For $\mathrm{B}_{\mathrm{T}}=8,9,10$ the detectable amplitude at the $99 \%$ confidence level is equal to $.06, .12$ and $.24 \mathrm{mag}$ respectively. All classical variables are indeed detectable by Tycho. A spectacular increase of the number of confirmed variables is expected, in particular among luminous stars and semi-regulars.

A major revision of our knowledge of the stellar instabilities is a foreseen by-product of the Hipparcos mission.

\section{ACKNOWLEDGMENTS}

We are indebted to $L$. Weber for the preparation of the colour plate.

\section{REFERENCES}

Burki, G., Kviz, Z., North, P. 1992, $A 8 A, 256,463$

Gray, D.F. 1991, in Angular Momentum Evolution of Young Stars, vol. 183,

Grenon, M. 1992, $A \mathcal{B} A, 258,88$

Harvey, J.W. 1988, in Advances in Helio- and Asteroseismology vol. 497

Mauder \& Høg. E. 1987, $A \& A, 185,349$

Mazzitelli, I. 1972, Mem. Soc. Astr. Ital., 45, 541

Mignard, F., Froschlé, M., Falin, J.L. 1992, $A \& A, 258,142$

North, P. 1992, private communication

Turon, C. et al. 1992, The Hipparcos Input Catalogue, Eds. ESA SP-1136

Waelkens, C. 1991, $A 8 A, 246,453$

Wielen, R. 1974, Highlights of Astronomy, 3, 395

Young, A.T. 1984, NASA Conf. Proc. 2350, p.8, Eds. W. Borucki and A. Young 